\title{
Identifying the Most Critical Intersections in Transportation Networks
}

\author{
Emre KUŞKAPAN* ${ }^{*}$ M. Yasin ÇODUR, Ahmet TORTUM
}

\begin{abstract}
With the increasing population worldwide, the number of vehicles is increasing day by day. This increase also creates different traffic problems. Various analysis methods are developed to solve traffic problems and to better examine the road transport network. Especially with the development of technology and intelligent transportation systems entering our lives, analyses can be made using computer software and programs. In this study; using the social network analysis method, the use of which has increased in the field of transportation in recent years, the highway network structure of Erzurum Province - which consists of district connection roads has been analyzed. While making the analysis, the network structure was examined through five different centrality concepts and the critical sequence of the intersections in the district connection roads was determined. Accuracy percentages of the concepts of centrality were determined by comparing the sequences obtained with the sequences actually applied. Then, it was determined that the most suitable centrality concept for the study was Bonacich Power.
\end{abstract}

Keywords: Highway network; Interdisciplinary sciences; Social network analysis; Traffic problems

\section{INTRODUCTION}

Along with the increasing number of vehicles in the world, various transportation problems have been occurring. Among transportation problems, the most common ones are traffic accidents, traffic congestions, air, and noise pollution. Observational solutions, which are the traditional methods being used to examine the transportation problems that occur and to offer solutions, have become insufficient in time. Thanks to the development of technology and Intelligent Transportation Systems (ITS); transportation problems are examined with multiple aspects and in more detail, rather than superficially. For this purpose, many transportation modelling and analysis methods have been developed. The developed modelling and analysis methods can be done more easily with computer programs. The numerical data being used for the application of the methods can be obtained by means of surveys, counting devices, cameras, etc.

In the existing literature, most researchers are using the computer program to solve transportation problems in studies [1-2]. These studies have also increased thanks to the provision of numerical data and the increase of methods used. One of the most preferred methods of solving transportation problems is Geographic Information System (GIS) based network analysis. With this method factors such as air pollution, traffic congestion, traffic accidents, noise pollution, and road deformations can be examined [3]. Another method used to examine traffic congestion is speed management. Traffic congestion: It can be modeled with software such as Vissim and Visum, which are simulation methods. By applying speed management analysis, these models can be determined for how the performance of the road network in the study area changes in terms of vehicle delays, total travel times and speed values [4]. Traffic impact analysis method is also used to determine the possible effects on general transportation and traffic congestions. The purpose of this method is not only to evaluate traffic but also to predict the status of future traffic. In this method, intersection design and Signal Coordination can also be done using simulation [5]. Wavelet transform method is also used in the analysis of traffic congestion. With this method, shock waves in highway traffic can be investigated. As the formation and propagation of shock waves are effective in the analysis of traffic congestion, the preferred state of the wavelet transform method is increasing. Using the cell transfer traffic flow model, travel requests, capacity restrictions, and possible traffic congestions can be examined. This method can be used to overcome the capacity reduction on the roads and to provide the maximum possible flow in traffic congestion situations [6]. Another method used to examine traffic congestion is the multi-class traffic flow model. This model can analyze traffic congestions by focusing on the movement of vehicles. In addition, speed profiles can be optimized for traffic congestion, energy consumption and travel time reduction performance by considering speed limits and traffic environment [7].

There are numerous methods of analysis regarding transport problems and also numerous studies conducted by those methods. Another method that has been added to these methods is the Social Network Analysis (SNA) method. Using this method all intersections in any network structure can be examined simultaneously. Using this feature, the most critical junctions in the network structure can be detected. In this way, the entire network structure is optimally utilized with the improvements made to the critical junctions determined in the network structure. Thus, traffic congestion is minimized.

Although the use of SNA in many different fields of study has been based on many years, it is possible to say that its use in transportation has come to the fore in recent years. As an example of those studies carried out in the field of transportation on this subject, Park and Yilmaz used SNA to examine road transport networks in various ways. They determined the concepts of centrality that were used in the analysis method as a method to study the selected road networks at the city, district or state levels. In the obtained results they stated that it would help to gather in-depth information about existing road networks for those who are experts in transportation [8]. In another study, Cheng et al. analyzed the Singapore metro network using SNA. They calculated some concepts of centrality to identify critical nodes in the network. They compared their results with the concepts of centrality based on traditional topology. They also proposed a new concept of centrality which also includes the delay in travel time [9]. Dörry and 
Decoville analyzed the need for cross-border public transport policies in metropolitan areas in Europe. With the data they obtained in various surveys, they proposed a flexible policy network by applying SNA on metropolitan cities and transportation policies in the Luxembourg region. They highlighted the need to integrate these flexible policy networks across Europe, with the declared economic, socio-cultural and spatial aspects [10]. In another study on this subject, Tse et al. considered people as sensors to monitor human activities in traffic. In this way, they suggested that valuable information can be obtained by applying data mining techniques to data created through social networks. A case study analyzing the relationship between weather conditions and traffic congestions was presented with this idea [11]. In another important study conducted in this field, El-adaway et al. investigated traffic congestions using SNA for three different areas in the US state of Louisiana. Different centrality concepts were calculated for the intersections in each case study. As a result of those calculations, the most critical intersections were identified. It was observed that the obtained critical intersections were just the same as the ones determined through technical investigations conducted by the ministry and improvement works being carried out [12]. Accordingly, SNA has proven to be an important method for analyzing and improving transportation networks $[13,14]$.

In this study, SNA method was applied to the highway network structure consisting of the county connection roads of Erzurum Province. Road network structure and traffic volume data were used while applying SNA method. According to this method, the order of the most critical intersections for five different centrality concepts (closeness, degree, betweenness, eigenvector and Bonacich power) was determined. A comparison was made between the determined order and the real order applied for speeding the flow of traffic. By determining the accuracy values of each centrality concept, the most appropriate centrality concept for the road network structure was determined.

\section{METHODOLOGY \\ 2.1 Study Area}

Erzurum Province was designated as the study area. It is in the north of Turkey's Eastern Anatolia Region and is located in a position that connects many cities. It is an important transition point in the region, especially due to the existence of the E-80 highway, defined as International E-Roads on the east-west axis [15]. It connects the provinces of Ardahan, Kars, Ağr1, Bayburt and Erzincan in the east-west direction. Also, it is connected to the East Black Sea region provinces Trabzon, Rize and Artvin in the north-south direction, by means of an increasing number of tunnels in recent years. Besides, it is connected to the provinces of Bingöl and Muş, by means of the roads in the south region, despite being used less, compared to other directions. In addition to those provincial connections, it consists of 20 districts, 3 of which are central districts [16]. Due to the fact that some connection roads between the districts are not very developed, various problems may occur in transportation. The most common of these disruptions is that the roads can be temporarily closed due to snowing and icing during the winter months. Since Erzurum is located in a harsh continental climate, an average of 149 days of frost per year is encountered [17]. There is a significant increase in traffic accidents caused by frequent occurrences of frost and snow in winter. In order to combat this situation, studies are carried out by public institutions. It is considered that this study will bring significant benefits, in terms of obtaining highly efficient results and minimizing transportation problems.

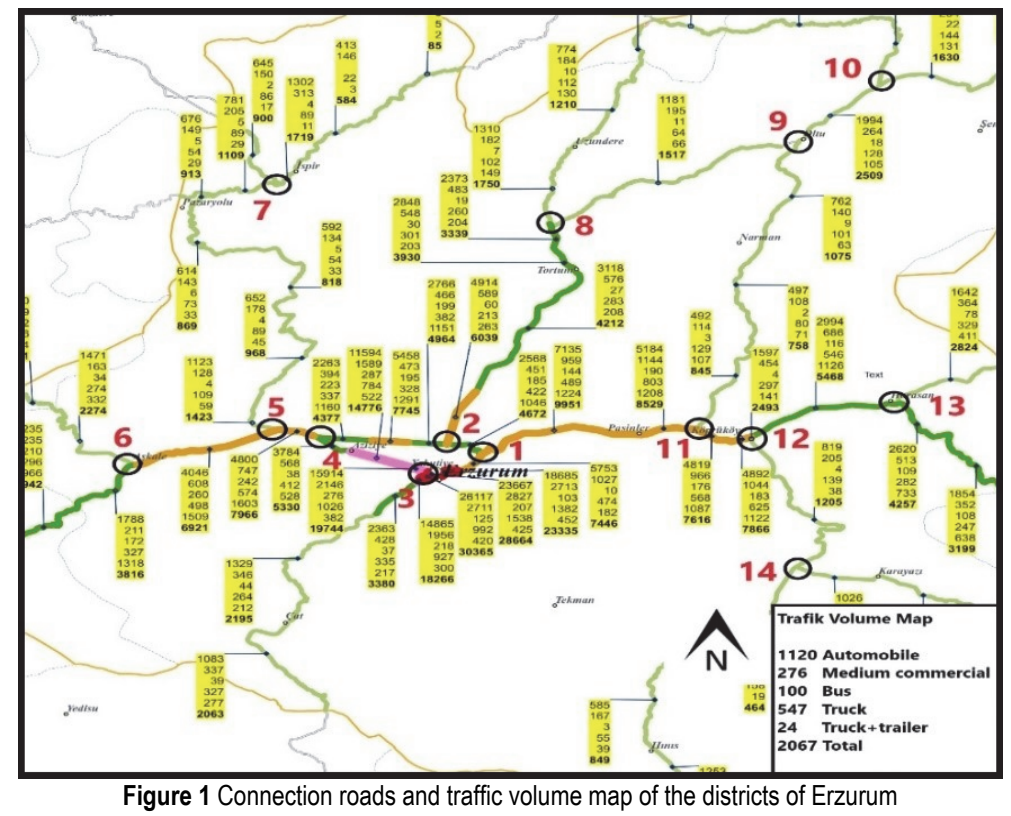

In this study, each of the Erzurum Province district connection roads has been identified as a connecting element (Fig. 1). In addition, the intersection points of these connection roads are expressed as node points. A total of 14 node points were obtained by enumerating each intersection. The numerical values in the connecting roads between the nodes represent the traffic volume data. Traffic volumes represent traffic in both directions over 24 hour period. This value is called the annual average daily 
traffic. Vehicle counts in traffic are obtained as a result of counts made by the General Directorate of Highways [18].

\subsection{Social Network Analysis}

SNA, which means the digitization of inter-object relations, is used to analyze the relationships of various organizations or their networks, political, formal-informal, family, geographic, transportation or any other form of relationships. In other words, network analysis enables it to examine how the elements, organizations, or systems within the network affect the way they work [19]. The advantage of SNA is that, unlike many other methods, it focuses on interaction (rather than individual behavior), and it is less time consuming, more economic and practical. In addition, it is an interdisciplinary analysis method which can be applied in many areas such as social networks, political networks, electricity networks, and transportation networks [20]. SNA, -especially when applied to transport networks - is very important for country policies because it is an indisputable fact that innovative strategies for transportation policies contribute to sustainable economic growth.

By using SNA the following can be determined:

- To what extent any node is connected within the network,

- The overall importance of any node in the network,

- To what extent any node is central,

- How data flows through the network can be determined [21].

The most important step for this method is the calculation of the centrality of nodes because the concept of centrality is the most basic concept forming the core of SNA. This statement focuses on the nodes in the network structure and tries to answer the question of how important the nodes are within the network. The higher the centrality of a node is in the network, the more critical is the node. With the criterion of centrality, a numerical value is assigned to all nodes in the network, and thanks to this value it is possible to compare and sort the nodes. In this study, the values of the five different centrality concepts were calculated.

\subsubsection{Closeness Centrality}

It is the indicator of the degree of closeness (or distance) of an actor (node) in the network to (or from) other actors, either directly or indirectly. Closeness is the sum of a node's shortest distances to other nodes in the network, and the ability to access information reflects how quickly an actor (node) can connect to other actors in the network. According to this concept of centrality, it is not necessary for a node to have a high degree in order to have a high closeness. Weakness or strength of the connections between nodes can be calculated by the measure of closeness centrality $[22,23]$.

Closeness centrality is defined by Eq. (1).

$C_{C}(i)=\left[\sum_{j=1}^{N} d(i, j)\right]^{-1}$

Normalized closeness centrality is defined by Eq. (2).

$$
C_{C}{ }^{\prime}(i)=\left(C_{C}(i) /(N-1)\right)
$$

where $d(i, j)$ represents the distance between vertices $i$ and $j ; N$ represents the number of nodes in the network.

\subsubsection{Degree Centrality}

Degree centrality is equal to the number of links (ties) of an actor to other actors. It not only reflects the connectivity of each node to other nodes but also relies on the network size. In other words, the larger the network, the higher the maximum degree of centralization [24]. Thus, a certain degree of centrality indicates that an actor either has many connections in a small network or has only a small number of links in a large network [25]. When the degree centrality of any node is calculated, the sum of the tie values to that node is calculated as shown in Eq. (3).

$C_{d}(i)=\sum_{j} X_{i j}$

$X$; actor. $i$ and $j$; nodes.

\subsubsection{Betweenness Centrality}

Betweenness centrality addresses the relationships and how those between the binary elements that are not directly connected are controlled or directed by other actors. It is also described as one step developed form of Closeness Centrality. Betweenness centrality is an important indicator of excessive information exchange within a network or control of the flow of resources. This measurement is based on the shortest number of ties passing through a node [26]. It is calculated by using Eq. (4) and Eq. (5).

While calculating this centrality, what is taken into consideration is whether a node is on the shortest road on the transition between other nodes. The important point is the fact that there may be more than one shortest distance between two nodes.

$C_{b}(i)=\sum_{j<k} g_{i j}(i) / g_{j k}$

It is usually normalized by:

$C_{b}{ }^{\prime}(i)=\left(C_{b}(i) /(n-1)(n-2) / 2\right)$

where $g_{j k}$ : the number of geodesics connecting $j k$, and $g_{j k}$ : the number that actor $i$ is on.

\subsubsection{Eigenvector Centrality}

It is defined as a more comprehensive and developed form of Degree Centrality. Eigenvector centrality shows the importance of a node in the network. The eigenvector center is not equal to all connections in the network; it assumes that effective nodes act on less efficient nodes to which they are associated [27]. It depends on the number of connections and the quality of the links. If a node has a small number of high-quality connections, it is more effective than a node with a large number of medium- 
quality connections [28]. For network $G=(V, E)$, with $\mid V$ as the number of vertices, let the adjacency matrix $\boldsymbol{A}=(a v$, $t) . M(v)$ be a set of neighbors of $v . a v, t=1$ if vertex $v$ is linked to vertex $t$, and $a v, t=0$ otherwise. $\lambda$ is a constant. The eigenvector centrality score of $v$ is obtained by Eq. (6).

$x_{v}=\frac{1}{\lambda} \sum_{t \in M(V)} x_{t}=\frac{1}{\lambda} \sum_{t \in G} a_{v, t} x_{t}$

\subsubsection{Bonacich Power Centrality}

It is a measure that determines node centrality based on the degree centrality of adjacent nodes, where a node's degree centrality is its summed connections to others, weighted by their centralities. This centrality depends not only on how many connections a node has but also on how many connections its neighbors have (and on how many connections its neighbors' neighbors have, and so on). Therefore, it investigates the network as a whole, both in terms of network structure and in terms of degree. The highest value found here represents the most powerful node on the network, as shown in Eq. (7) [29].

$$
C(\alpha, \beta)=\alpha(I-\beta R)^{-1} R I
$$

$\boldsymbol{R}$ is the adjacency matrix (can be valued), $\boldsymbol{\beta}$ reflects the extent to which you weigh the centrality of nodes tied to the studied node. $\boldsymbol{I}$ is the identity matrix, $\boldsymbol{\alpha}$ is a scaling vector, $\boldsymbol{l}$ is a matrix of all ones.

Ucinet program is one of the most common programs used in SNA to calculate the values of centrality, to model the network structure and to analyze the network. This program is a comprehensive package that can read and write text files in various formats and also excel files. Also, it has features like network modeling, centering measures, matrix analysis and statistical analysis based on permutation [30]. Netdraw program, which is used to draw diagrams of social networks, is also included in this package. In this way, no extra program is required to create network schemes. For this reason, in this study modeling and analyses were done using Ucinet and Netdraw programs.

\section{RESULTS}

After determining the nodes forming the network structure, an adjacency matrix was created which indicates the transition state between these nodes. By means of this matrix, the transition between node points is digitized. In the case of a direct transition state between any two nodes, the adjacency matrix is obtained by writing a value of 1 and a value of 0 is written if there is no transition state [31]. An adjacency matrix is given in Tab. 1, which indicates the transition situation between 14 node points.

In the adjacency matrix obtained, the traffic volume values between the nodes are placed where the value 1 is written. After uploading the new matrix to the Ucinet program, the network structure model was created.
Table 1 Adjacency matrix in the study area

\begin{tabular}{|c|c|c|c|c|c|c|c|c|c|c|c|c|c|c|}
\hline & 1 & 2 & 3 & 4 & 5 & 6 & 7 & 8 & 9 & 10 & 11 & 12 & 13 & 14 \\
\hline 1 & 0 & 1 & 1 & 0 & 0 & 0 & 0 & 0 & 0 & 0 & 1 & 0 & 0 & 0 \\
\hline 2 & 1 & 0 & 0 & 1 & 0 & 0 & 0 & 1 & 0 & 0 & 0 & 0 & 0 & 0 \\
\hline 3 & 1 & 0 & 0 & 1 & 0 & 0 & 0 & 0 & 0 & 0 & 0 & 0 & 0 & 0 \\
\hline 4 & 0 & 1 & 1 & 0 & 1 & 0 & 0 & 0 & 0 & 0 & 0 & 0 & 0 & 0 \\
\hline 5 & 0 & 0 & 0 & 1 & 0 & 1 & 1 & 0 & 0 & 0 & 0 & 0 & 0 & 0 \\
\hline 6 & 0 & 0 & 0 & 0 & 1 & 0 & 0 & 0 & 0 & 0 & 0 & 0 & 0 & 0 \\
\hline 7 & 0 & 0 & 0 & 0 & 1 & 0 & 0 & 0 & 0 & 0 & 0 & 0 & 0 & 0 \\
\hline 8 & 0 & 1 & 0 & 0 & 0 & 0 & 0 & 0 & 1 & 1 & 0 & 0 & 0 & 0 \\
\hline 9 & 0 & 0 & 0 & 0 & 0 & 0 & 0 & 1 & 0 & 1 & 1 & 0 & 0 & 0 \\
\hline 10 & 0 & 0 & 0 & 0 & 0 & 0 & 0 & 1 & 1 & 0 & 0 & 0 & 0 & 0 \\
\hline 11 & 1 & 0 & 0 & 0 & 0 & 0 & 0 & 0 & 1 & 0 & 0 & 1 & 0 & 0 \\
\hline 12 & 0 & 0 & 0 & 0 & 0 & 0 & 0 & 0 & 0 & 0 & 1 & 0 & 1 & 1 \\
\hline 13 & 0 & 0 & 0 & 0 & 0 & 0 & 0 & 0 & 0 & 0 & 0 & 1 & 0 & 0 \\
\hline 14 & 0 & 0 & 0 & 0 & 0 & 0 & 0 & 0 & 0 & 0 & 0 & 1 & 0 & 0 \\
\hline
\end{tabular}

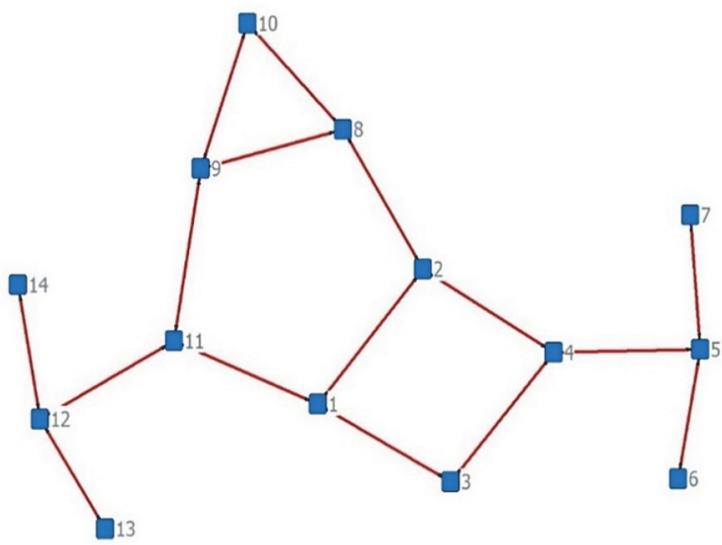

Figure 2 Modelling of the network structure

Fig. 2 shows the road network structure model created with the locations of the node points. After the model is created, it becomes possible to examine the centrality values, which are the most determining criteria for SNA. Each of the centrality values examines the network structure from different directions. Accordingly, the status of the network structure and what kind of data it consists of are very important in terms of centrality [32]. Depending on the state and content of the network structures, whether or not the concepts of centrality are suitable for that network structure also differ. For example, for a network structure, the locations of the node points are of no importance, while the tie values of another network structure may have no significance. Therefore, the best concept of centrality for a certain network should be determined by analysing the network structure with the concept of more than one centrality. In the closeness centrality, which is the first concept of centrality, the tie values in the network structure are not important, whereas the locations of the nodes in the network are important. Therefore, the closeness centrality values of the nodes, which are positioned at the mid-points in the road network are expected to be higher. When calculating this centrality, the shortest geodetic distances must be determined correctly, since there may be more than one route between two nodes. 
In order to make this process easy, it is necessary to create a closeness matrix. In Tab. 2, the closeness matrix based on the transition between each other has been created for 14 nodes in the network structure.

Table 2 Closeness matrix

\begin{tabular}{|c|c|c|c|c|c|c|c|c|c|c|c|c|c|c|}
\hline & 1 & 2 & 3 & 4 & 5 & 6 & 7 & 8 & 9 & 10 & 11 & 12 & 13 & 14 \\
\hline 1 & 0 & 1 & 1 & 2 & 3 & 4 & 4 & 2 & 2 & 3 & 1 & 2 & 3 & 3 \\
\hline 2 & 1 & 0 & 2 & 1 & 2 & 3 & 3 & 1 & 2 & 2 & 2 & 3 & 4 & 4 \\
\hline 3 & 1 & 2 & 0 & 1 & 2 & 3 & 3 & 3 & 3 & 4 & 2 & 3 & 4 & 4 \\
\hline 4 & 2 & 1 & 1 & 0 & 1 & 2 & 2 & 2 & 3 & 3 & 3 & 4 & 5 & 5 \\
\hline 5 & 3 & 2 & 2 & 1 & 0 & 1 & 1 & 3 & 4 & 4 & 4 & 5 & 6 & 6 \\
\hline 6 & 4 & 3 & 3 & 2 & 1 & 0 & 2 & 4 & 5 & 5 & 5 & 6 & 7 & 7 \\
\hline 7 & 4 & 3 & 3 & 2 & 1 & 2 & 0 & 4 & 5 & 5 & 5 & 6 & 7 & 7 \\
\hline 8 & 2 & 1 & 3 & 2 & 3 & 4 & 4 & 0 & 1 & 1 & 2 & 3 & 4 & 4 \\
\hline 9 & 2 & 2 & 3 & 3 & 4 & 5 & 5 & 1 & 0 & 1 & 1 & 2 & 3 & 3 \\
\hline 10 & 3 & 2 & 4 & 3 & 4 & 5 & 5 & 1 & 1 & 0 & 2 & 3 & 4 & 4 \\
\hline 11 & 1 & 2 & 2 & 3 & 4 & 5 & 5 & 2 & 1 & 2 & 0 & 1 & 2 & 2 \\
\hline 12 & 2 & 3 & 3 & 4 & 5 & 6 & 6 & 3 & 2 & 3 & 1 & 0 & 1 & 1 \\
\hline 13 & 3 & 4 & 4 & 5 & 6 & 7 & 7 & 4 & 3 & 4 & 2 & 1 & 0 & 2 \\
\hline 14 & 3 & 4 & 4 & 5 & 6 & 7 & 7 & 4 & 3 & 4 & 2 & 1 & 2 & 0 \\
\hline
\end{tabular}

When the values in the obtained closeness matrix are examined, because the nodes in the middle regions of the network structure are easy to access to other nodes, their closeness values are also high. However, since the nodes in the edge areas in the network structure are more difficult to reach to other nodes in the edge areas, the closeness value is lower. In the degree centrality, while calculating the centrality values, for each node, the sum of the tie values in that node in the network is calculated. Contrary to closeness centrality, the important thing is the tie values, not the location of the nodes in the network structure. In addition, the more ties attached to a node, the more advantage it provides to the degree centrality in that node. Like closeness centrality, betweenness centrality is a shortest-path-based centrality concept. The difference is that betweenness centrality is about the location of a node between the shortest roads, rather than being its closeness to the other nodes. For example, when calculating the betweenness centrality of a node, first the shortest distances between the other nodes are calculated, and then how many of them pass through that node are determined [33]. The more a node takes part in the shortest paths, the higher its betweenness centrality value is. The most important factor to consider while calculating the betweenness centrality of the nodes is that there may be more than one shortest path between the nodes.

Eigenvector centrality is a measurement of centrality that takes degree centrality a step further. In this concept, the importance of a node depends not only on the number of its neighbors it has, but also depends on the highness of tie values of its neighbors. In this way, eigenvector centrality can yield results that reflect the network structure more accurately than degree centrality. As for the Bonacich power centrality concept, which was finally investigated, both the tie values and neighborhoods of nodes in the network are being taken into account. While taking the neighbourhoods into account, all the node points in the network are being taken into consideration. Therefore, it investigates the network structure more comprehensively, compared to eigenvector centrality. This makes Bonacich power centrality different from eigenvector centrality [34].
Table 3 Centrality values of the nodes in the network structure

\begin{tabular}{|c|c|c|c|c|c|}
\hline Node & Clos. & Degree & Between. & Eigen. & Bonacich \\
\hline 1 & 0.42 & 23986 & 53333 & 0.54 & 6512943 \\
\hline 2 & 0.43 & 15806 & 50333 & 0.16 & 2689663 \\
\hline 3 & 0.37 & 41826 & 20 & 0.65 & 3878942 \\
\hline 4 & 0.38 & 17029 & 62667 & 0.48 & 5156097 \\
\hline 5 & 0.31 & 16490 & 46 & 0.12 & 2235251 \\
\hline 6 & 0.24 & 7269 & 0 & 0.03 & 787990 \\
\hline 7 & 0.24 & 965 & 0 & 0.01 & 152091 \\
\hline 8 & 0.38 & 6529 & 22333 & 0.03 & 782657 \\
\hline 9 & 0.37 & 4720 & 17667 & 0.01 & 223951 \\
\hline 10 & 0.32 & 3235 & 0 & 0.01 & 96643 \\
\hline 11 & 0.41 & 17257 & 65667 & 0.20 & 4008246 \\
\hline 12 & 0.33 & 14548 & 46 & 0.05 & 1628404 \\
\hline 13 & 0.25 & 5590 & 0 & 0.01 & 442980 \\
\hline 14 & 0.25 & 1206 & 0 & 0.01 & 95569 \\
\hline
\end{tabular}

The centrality values calculated for all nodes are shown in Tab. 3. When the values are examined, the node 2 which has the highest value for the closeness centrality value is located in the middle sections in the network structure. Similarly, the closeness centrality values of nodes such as 1-11-4 and 8, which are also close to the midpoints in the network structure, are higher, while the closeness centrality values of the edge nodes 6-7-13 and 14 are lower. In the concept of degree centrality, since the values of the tie values that are connected only to the nodes are important, intersections with high traffic volume are expected to have a high degree of centrality. In accordance with this situation, it is seen that the highest degree of centrality is at the 3-1-11 and 4 nodes, respectively. Node points with low traffic volumes are at the bottom of the ranking.

As for the values of betweenness centrality, since the shortest distance between the nodes is effective, it has a value of 0 at the endpoints. The nodes 11 and 4 have a high value since they are in an important position in the transition to the edge nodes and they have no alternative. For example, considering the nodes 12-13-14, when a transition takes place to those nodes from another node, it is a must that this transition happens via node 11 . Then this situation causes the betweenness value of node 11 to be high. When the values of eigenvector centrality are analyzed, it is seen that 3-1-4-11 and 2 nodes have higher values. The high traffic volumes of these points and their neighboring points with high traffic volumes cause this situation. In the Bonacich power concept of centrality, which was last examined, it is seen that the highest values emerge at node points 1-4-11 and 3, respectively. In this concept, such a result emerges because both traffic volume data and road network structure are effective. Although the traffic volume values of the intersection 3 are higher than the intersections 1-4 and 11, they caused them to be lower in the ranking due to being on the edge of the network. Node points 14 and 10, which have the lowest Bonacich power values, are effective both on the edge of the network structure and having low volume values. 


\section{DISCUSSION}

It is necessary to compare the calculated values with the real values in order to determine the concept that gives the most accurate results for the road network structure from five centrality concepts. In the snow-fighting works conducted by Erzurum Metropolitan Municipality in the winter, the critical order of the intersections and the SNA centrality criteria were compared in Fig. 3 and Fig. 4.

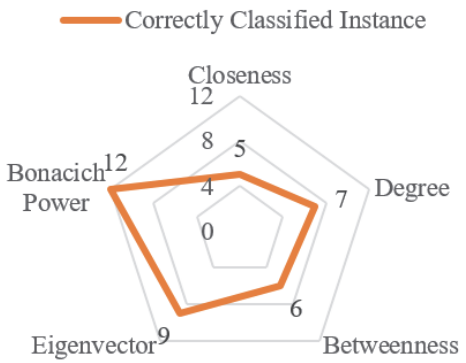

Figure 3 The correctly classified instance of the centrality concepts

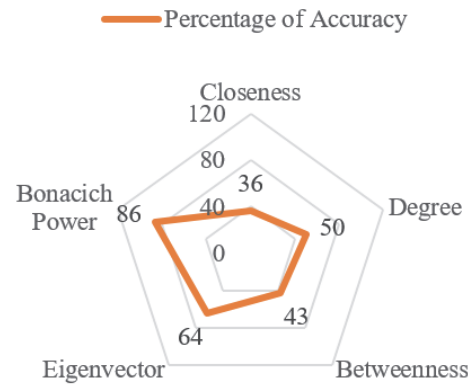

Figure 4 The accuracy percentage of the centrality concepts

When the results in the graphs are analyzed, it can be seen that Bonacich power centrality gives more accurate values in the critical classification of intersections. In this context, it yielded much better results than other centrality criteria with $86 \%$ accuracy. The concept of closeness, degree, and betweenness which evaluate the network structure only by the traffic volume value or the locations of the intersection points have a lower degree of accuracy. Eigenvector and Bonacich power, which are among the concepts of centrality in SNA, and which are examined in the study, are based on both criteria [35]. However, eigenvector centrality has a different structure than Bonacich power centrality, because it only takes into consideration the tie values of its neighboring node points. Bonacich power centrality takes into account all the neighborhoods of nodes as well as the network structure. Therefore, it is seen that the order of Bonacich power centrality concept is similar to real values. Bonacich Power centrality takes into account both the network structure and the values of the nodal points due to its structure. Therefore, it can be used as an important determinant in the analysis of future transportation networks.

\section{CONCLUSION}

In this study, a transportation network consisting of the connecting roads of the districts of Erzurum Province was analyzed, using SNA. The main purpose is to speed up the traffic flow by quickly solving the problems in the transportation network. To this end, results were obtained by means of five different centrality concepts by using the transition status of the connection roads of the determined transportation network, the location of the intersection points and traffic volume data. As a result of the analysis, it was determined that the concept of centrality, which is the most suitable for the road network structure, is the Bonacich power. It was determined that the ranking of the concept of Bonacich power centrality and the ranking applied in reality are close to each other. Therefore, the priority order of improvement in road network structure should be applied to intersections with the highest value in the centrality of Bonacich power, respectively. If this priority order is done correctly, traffic accidents and flow failures due to snow and frost events will be reduced in winter. In this context, thanks to the SNA method, various transportation networks can be analyzed and improved at the right points and the order of priority can be determined, thus affecting the entire network structure positively. For all these reasons, the SNA method should become widespread in transportation planning and the application area should be expanded. With this study, it is predicted that the analysis of transportation networks will become more practical in the future. The method of the study can be applied especially in cities with high traffic density and need improvement at intersection points.

\section{REFERENCES}

[1] Saha, P. \& Ksaibati, K. (2016). An optimization model for improving highway safety. Journal of traffic and transportation engineering (English edition),3(6), 549-558. https://doi.org/10.1016/j.jtte.2016.01.004

[2] Ismail, K. \& Sayed, T. (2012). Risk-optimal highway design: Methodology and case studies. Safety science, 50(7), 1513-1521. https://doi.org/10.1016/j.ssci.2012.02.001

[3] Beuthe, M., Degrandsart, F., Geerts, J. F., \& Jourquin, B. (2002). External costs of the Belgian interurban freight traffic: a network analysis of their internalisation. Transportation Research Part D: Transport and Environment, 7(4), 285-301. https://doi.org/10.1016/S1361-9209(01)00025-6

[4] Ponnurangam, P. \& Umadevi, G. (2016). Traffic Impact Analysis (TIA) for Chennai IT Corridor. Transportation Research Procedia, 17, 234-243. https://doi.org/10.1016/j.trpro.2016.11.079

[5] Şahin, İ., Kuşakci, S. Ş., \& Aydin, G. (2016). Shock Waves in Highway Traffic: Macroscopic and Microscopic Investigation with Wavelet Transform. Teknik Dergi, 27(2), 7429-7452.

[6] Zhang, Y. \& Ioannou, P. A. (2018). Stability analysis and variable speed limit control of a traffic flow model. Transportation Research Part B: Methodological, 118, 3165. https://doi.org/10.1016/j.trb.2018.10.005

[7] Bede, Z., Németh, B., \& Gáspár, P. (2017). Modeling and Simulation Based Analysis of Multi-Class Traffic with Look-Ahead Controlled Vehicles. Transportation Research Procedia, 27, 593-599. https://doi.org/10.1016/j.trpro.2017.12.125

[8] Park, K. \& Yilmaz, A. (2010). A social network analysis approach to analyze road networks. ASPRS Annual Conference. San Diego, CA, 1-6.

[9] Cheng, Y. Y., Lee, R. K. W., Lim, E. P., \& Zhu, F. (2015). Measuring centralities for transportation networks beyond structures. Applications of social media and social network analysis, 23-39. https://doi.org/10.1007/978-3-319-19003-7_2

[10] Dörry, S. \& Decoville, A. (2016). Governance and transportation policy networks in the cross-border 
metropolitan region of Luxembourg: A social network analysis. European Urban and Regional Studies, 23(1), 6985. https://doi.org/10.1177/0969776413490528

[11] Tse, R., Zhang, L. F., Lei, P., \& Pau, G. (2018). Social network based crowd sensing for intelligent transportation and climate applications. Mobile Networks and Applications, 23(1), 177-183. https://doi.org/10.1007/s11036-017-0832-y

[12] El-Adaway, I. H., Abotaleb, I., \& Vechan, E. (2018). Identifying the most critical transportation intersections using social network analysis. Transportation planning and technology, 41(4), 353-374. https://doi.org/10.1080/03081060.2018.1453456

[13] El-Adaway, I. H., Abotaleb, I. S., \& Vechan, E. (2017). Social network analysis approach for improved transportation planning. Journal of Infrastructure Systems, 23(2), 05016004. https://doi.org/10.1061/(ASCE)IS.1943-555X.0000331

[14] El-adaway, I. (2014). Analyzing traffic layout using dynamic social network analysis.

[15] See Republic of Turkey General Directorate of Highways, International E-Roads. (2019). https://www.kgm.gov.tr/Sa yfalar/KGM/Site Eng/Root/MainPageEnglish.aspx

[16] See Erzurum Provincial Culture and Tourism Directorate, Erzurum Districts. (2019). https://erzurum.ktb.gov.tr/TR56045/ilceler.html

[17] Koşan, Z., Kavuncuoğlu, D., Çalikoğlu, E. O., \& Yerli, E. B. (2018). Evaluation of air pollution by PM10 and SO2 levels in Erzurum province, Turkey: Descriptive study. Journal of Surgery and Medicine, 2(3), 265-268. https://doi.org/10.28982/josam.422921

[18] See General Directorate of Highways, 2017 Traffic and Transport information. (2017). https://www.kgm.gov.tr/ SiteCollectionDocuments/KGMdocuments/Istatistikler/Traf ikveUlasimBilgileri/17TrafikUlasimBilgileri.pdf.

[19] Wasserman, S. \& Faust, K. (1994). Social network analysis: Methods and applications, 8. Cambridge university press. https://doi.org/10.1017/CBO9780511815478

[20] Ağcasulu, H. (2018). A Method Analyzing Relationships in Social Sciences: Social Network Analysis. Journal of Atatürk University Institue Social Science, 22, 1915-1933.

[21] Freeman, L. C. (2014). A Study in the Sociology of Science.

[22] Agneessens, F., Borgatti, S. P., \& Everett, M. G. (2017). Geodesic based centrality: Unifying the local and the global. Social Networks, 49, 12-26. https://doi.org/10.1016/j.socnet.2016.09.005

[23] Du, D. (2019). Social network analysis: Centrality measures. University of New Brunswick.

[24] Opsahl, T., Agneessens, F., \& Skvoretz, J. (2010). Node centrality in weighted networks: Generalizing degree and shortest paths. Social networks, 32(3), 245-251. https://doi.org/10.1016/j.socnet.2010.03.006

[25] Gonçalves, J. A. M., da Silva Portugal, L., \& Nassi, C. D. (2009). Centrality indicators as an instrument to evaluate the integration of urban equipment in the area of influence of a rail corridor. Transportation Research Part A: Policy and Practice, 43(1), 13-25. https://doi.org/10.1016/j.tra.2008.06.010

[26] Brandes, U. (2005). Network analysis: methodological foundations, 3418. Springer Science \& Business Media.

[27] Ando, H., Bell, M., Kurauchi F., Wong, K. I., Cheung, K. (2020). Connectivity evaluation of large road network by capacity-weighted eigenvector centrality analysis. Transportmetrica A: Transport Science, 1-27. https://doi.org/10.1080/23249935.2020.1804480

[28] Hagan, R., Feng, Y., \& Bush, J. (2015). Centrality Metrics. University of Tennessee, Knoxville.

[29] Bonacich, P. (2007). Some unique properties of eigenvector centrality. Social networks, 29(4), 555-564. https://doi.org/10.1016/j.socnet.2007.04.002
[30] Boyd, J. P., Fitzgerald, W. J., \& Beck, R. J. (2006). Computing core/periphery structures and permutation tests for social relations data. Social networks, 28(2), 165-178. https://doi.org/10.1016/j.socnet.2005.06.003

[31] Tunal1, V. (2018). Introduction to Social Network Analysis Book, Ankara,

[32] Hart, M. G., Ypma, R. J., Romero-Garcia, R., Price, S. J., \& Suckling, J. (2016). Graph theory analysis of complex brain networks: new concepts in brain mapping applied to neurosurgery. Journal of neurosurgery, 124(6), 1665-1678. https://doi.org/10.3171/2015.4.JNS142683

[33] Leydesdorff, L. (2007). Betweenness centrality as an indicator of the interdisciplinarity of scientific journals. Journal of the American Society for Information Science and Technology, 58(9), 1303-1319. https://doi.org/10.1002/asi.20614

[34] Faust, K. (1997). Centrality in affiliation networks. Social networks, 19(2), 157-191. https://doi.org/10.1016/S0378-8733(96)00300-0

[35] Watts, R. \& Witham, A. (2012). Social network analysis of sustainable transportation organizations (No. UVM TRC Report\# 12-008). University of Vermont. Transportation Research Center.

\section{Contact information}

\section{Emre KUŞKAPAN}

(Corresponding author)

Faculty of Engineering and Architecture,

Department of Civil Engineering/Transportation, Erzurum Technical University, Erzurum, Turkey

E-mail: emre.kuskapan@erzurum.edu.tr

\section{Yasin CODUR}

Faculty of Engineering and Architecture,

Department of Civil Engineering/Transportation, Erzurum Technical University,

Erzurum, Turkey

E-mail: mycodur@erzurum.edu.tr

\section{Ahmet TORTUM}

Faculty of Engineering

Department of Civil Engineering/Transportation, Ataturk University,

Erzurum Turkey

E-mail: atortum@atauni.edu.tr 